\title{
Enhanced particle transport caused by resonant magnetic perturbations in the
}

\section{J-TEXT tokamak}

Qiming Hu ${ }^{1}$, Ge Zhuang ${ }^{1}{ }^{*}$, Q. Yu ${ }^{2}$, Bo Rao ${ }^{1}, \mathrm{Li} \mathrm{Gao}^{1}$, Nengchao Wang ${ }^{1}$, Wei Jin ${ }^{1}$, Bin Yi ${ }^{1}$, Wubing Zeng $^{1}$, Wei Chen ${ }^{1}$, Yonghua Ding ${ }^{1}$, Zhipeng Chen $^{1}$, Xiwei $\mathrm{Hu}^{1}$ and J-TEXT Team ${ }^{1}$

1 State Key Laboratory of Advanced Electromagnetic Engineering and Technology, Huazhong University of Science and Technology, Wuhan, 430074, China

2 Max-Planck-Institut für Plasmaphysik, EURATOM association, 85748 Garching, Germany

* Corresponding author: ge-zhuang@mail.hust.edu.cn

\begin{abstract}
The effect of resonant magnetic perturbations (RMPs) on particle transport has been studied in J-TEXT tokamak. It is found that for the discharges with an existing saturated $2 / 1$ resistive tearing mode, applied RMPs of moderate amplitude lead to a decrease in electron density with a relative amplitude $3 \%<\left|\Delta n_{\mathrm{e}} / n_{\mathrm{e} 0}\right|<10 \%$ in plasma core, and the mode stabilization and electron temperature increase are observed simultaneously in this case. Sufficiently large amplitude of RMPs, however, lead to locked modes and much larger decrease in the electron density as well as in the electron temperature, with $\left|\Delta n_{\mathrm{e}} / n_{\mathrm{e} 0}\right| \sim 20 \%$. For the discharges without 2/1 tearing modes, applied RMPs cause a relative density decrease $\left|\Delta n_{\mathrm{e}} / n_{\mathrm{e} 0}\right| \sim 10 \%$ (30\%) before (after) field penetration. Using the two-fluid equations and experimental parameters as input, the numerical results approximately agree with experimental observations.
\end{abstract}

\section{Introduction}

In recent years, the resonant magnetic perturbations (RMPs) have been utilized for active control of the edge-localized modes (ELMs) [1-6] and for studying their effect on plasma confinement [7, 8] and magnetohydrodynamic (MHD) stabilities [9, 10]. It has often been observed in these experiments that there is a decrease in the electron density caused by applied RMPs (the so called density pump-out). An increased particle confinement was sometimes also observed in other experiments $[7,8]$. An understanding of the mechanism leading to the changed particle confinement is necessary. These phenomena have been explained in terms of the formation of stochastic magnetic fields in the tokamak edge region by RMPs [11-14], which could affect the radial electric field and the particle transport caused by plasma turbulence. However, the stochastic magnetic field is obtained in calculations under the vacuum field approximation. Taking into account the plasma response to the externally applied RMPs, the RMP amplitude inside the plasma could be considerably reduced compared to that obtained with the vacuum approximation. The screening current induced by plasma flow [15-22] and the rotation braking indicate the plasma response to applied RMPs [3, 9, 23-26].

Based on two-fluid equations, another mechanism affecting the particle transport by a single helicity of RMP is revealed [16, 27]. Depending on the frequency and direction of plasma rotation, the RMP can either increase or decrease the plasma density gradient around the corresponding rational surface [16,27]. The screening current, created by the interaction of the RMP with the rotating plasma, is mainly responsible for the additional radial particle fluxes. Similar results are 
also given in Ref. [17] for H-mode plasmas.

The J-TEXT tokamak [28] has been equipped with RMPs coils in order to study the plasma response to RMPs $[29,30]$. The applied RMP has been used to study its effect on $m / n=2 / 1$ tearing mode (TM) [31-33], and the suppression of 2/1 TM by it has often been observed [31, 32]. Different spectra and amplitude of RMPs generated by these coils allow studying the mechanism that lead to the changed particle confinement caused by RMPs.

In this paper, the experimental results about the effect of RMPs on particle confinement, observed in J-TEXT discharges, are presented. The experimental results show that the $m / n=2 / 1$ resonant component of RMPs is responsible for the observed decrease in the electron density. Here, $m$ and $n$ are the poloidal and toroidal mode numbers, respectively. In order to understand the experimental results, numerical calculations have also been carried out. With experimental parameters as input, the numerical results approximately agree with experimental observations.

This paper is organized as follows: in section 2 the experimental setup and results are described. The numerical results and comparisons with experimental ones are given in section 3. Finally, the results are discussed and summarized in section 4.

\section{Experimental setup and results}

\subsection{Experimental setup}

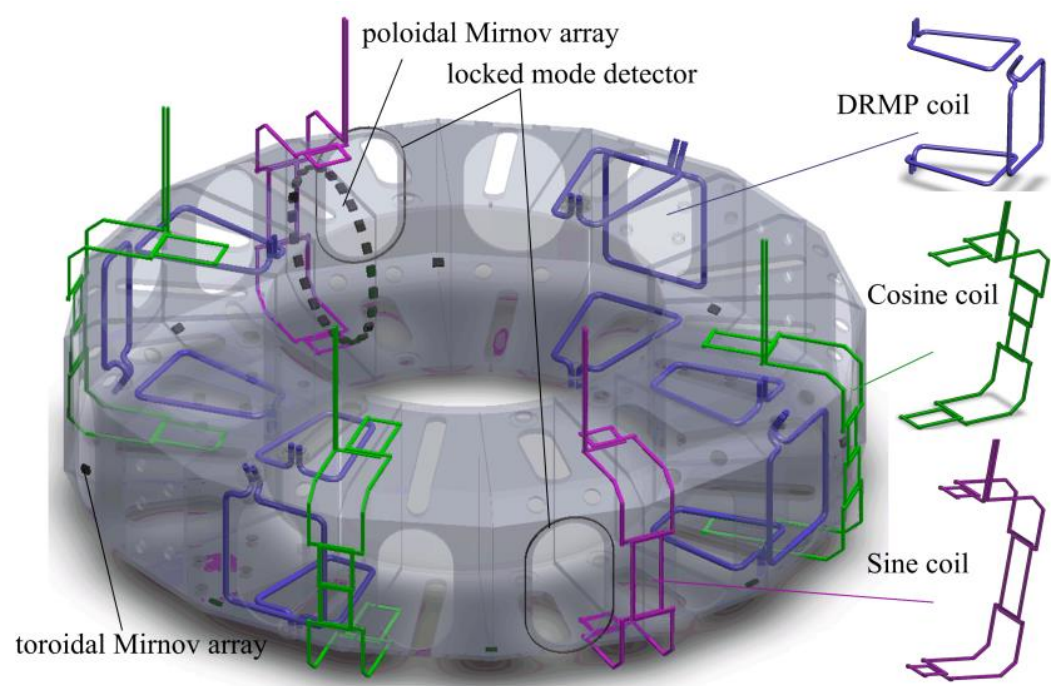

Figure 1. Layout of SRMP and DRMP coils in J-TEXT. SRMP consists of 3 cosine coils and 2 sine coils. DRMP consists of 12 saddle coils which are divided into 4 groups and located at 4 toroidal locations. The coil structure is shown on the right side. The SRMP coils are installed outside the vacuum vessel, while the DRMP coils are installed inside the vacuum vessel. The layout of poloidal Mirnov array, toroidal Mirnov array and locked mode detector are also shown in Ref. [34].

$\mathrm{J}$-TEXT is a circular, medium sized, limiter tokamak with a major radius $\left(R_{0}\right)$ of $1.05 \mathrm{~m}$ and a

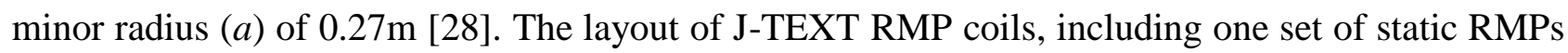
coils (SRMP) [29] and another set of dynamic RMPs (DRMP) [30], is shown in figure 1 [34]. The SRMP consists of 3 cosine coils and 2 sine coils distributed at five toroidal locations, and a detailed view of their structures is given on the right side of figure 1. The SRMP coils are installed outside the vacuum vessel at a minor radius of $r=0.39 \mathrm{~m}$. The three cosine coils are installed with a toroidal interval of $90^{\circ}$, and the two sine coils are placed opposite to each other. These five coils are 
connected in series and fed with a DC power supply, and the current direction of each coil can be controlled separately, including being bypassed. Because of the spatial structure and flexible connection of SRMP, the magnetic field can have different spatial phases, allowing the measurement of intrinsic error field to be possible [29]. The DRMP consists of 12 saddle coils which are divided into 4 groups and equipped at 4 toroidal locations, as also shown in figure 1, and the structure of a single group of coils is given at the right side of figure 1. The DRMP coils are installed inside the vacuum vessel [35].

In order to study the effect of RMPs on particle transport, three types of RMP spectra have been utilized in experiments: (a) Case A: The spectrum is generated by SRMP and dominated by the $m / n=2 / 1$ and $3 / 1$ components. In figure $2(a)$ the radial component of the RMP amplitude, $b^{1}{ }_{\mathrm{m}, \mathrm{n}}$, obtained for the vacuum approximation and for SRMP supplied current $I_{c}=1 \mathrm{kA}$, is shown as a function of poloidal mode number $(m)$ and normalized radius $(r / a)$ for the $n=1$ component. Superimposed on the spectra are the location of the $q=m / 1$ rational surfaces. The $2 / 1$ component at the plasma edge is $b_{2,1}^{1}(r=a)=0.63$ Gauss per kilo ampere. (b) Case $\boldsymbol{B}$ : The spectrum as shown in figure $2(b)$ is generated by SRMP and dominated only by the $m / n=3 / 1$ component. (c) Case $\boldsymbol{C}$ : The spectrum generated by the DRMP as shown in figure $2(c)$, has only a dominant $m / n=2 / 1$ component, and its strength at the plasma edge is $b^{1}{ }_{2,1}(r=a)=2.5$ Gauss per kilo ampere.
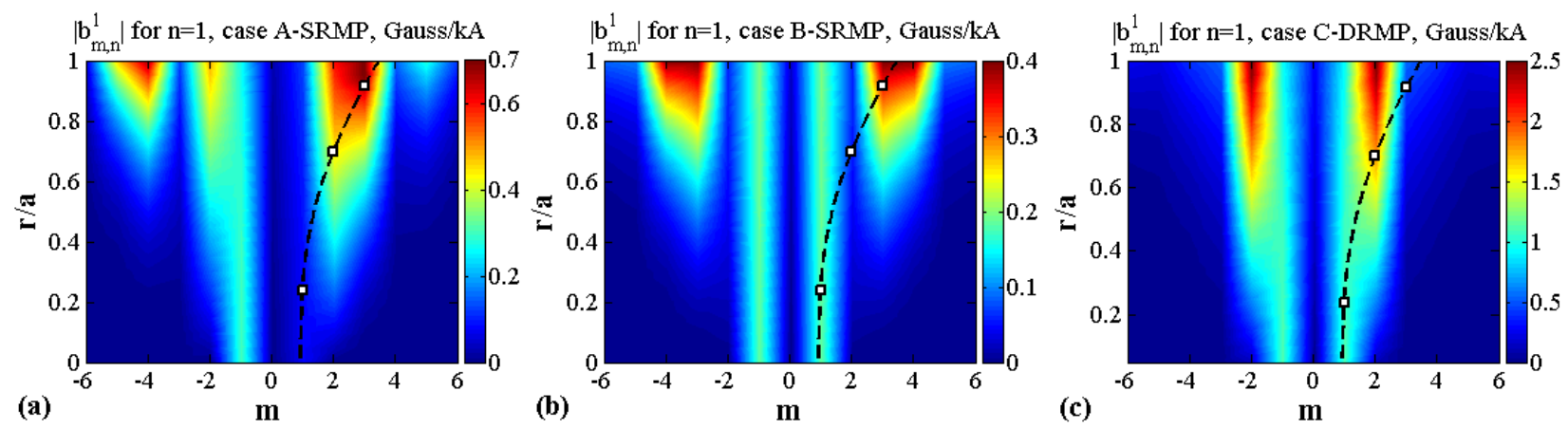

Figure 2. Poloidal spectrum of RMPs with $n=1$ for (a) Case $A$ generated by SRMP, (b) Case $B$ generated by SRMP and (c) Case $C$ generated by DRMP. Superimposed as squares and dashed curve are the $q=m / 1$ rational surfaces.

In experiments, the electron density is measured with seven-channel far-infrared (FIR) HCN interferometer viewing the plasma vertically with its impact parameters $R=1.0,1.05,1.1,1.15,1.2$, 1.25 and $1.3 \mathrm{~m}$ [36]. The electron density provided by the channel at $R=1.05 \mathrm{~m}$ is used to interpret the experimental results in the context. It will be noted in the text where more channels of density are needed in order to do the comparison. The MHD activity is measured by both poloidal and toroidal Mirnov arrays as shown in figure 1, and the locked mode detectors consist of two saddle coils installed at two opposite toroidal locations outside the vacuum vessel. Eight arrays of soft X-ray (SXR) are installed, and each array contains 16 viewing chords covering the plasma cross-section.

\subsection{Experimental results}

In order to study the resonant effects of RMP on particle transport, the experiments have been carried out in two forms. In the first, SRMP is applied to discharges with an initially saturated 2/1 TM. In the second, DRMP is applied to discharges with and without an initially 2/1 TM.

For the experimental results described below, the plasma parameters are as follows unless 
otherwise noted: plasma current is $I_{\mathrm{p}}=175 \mathrm{kA}$, toroidal magnetic field $B_{\mathrm{t}}=1.74 \mathrm{~T}$, the safety factor $q$ $=3.5$ at the plasma edge, and the $q=2$ surface is at about $r / a=0.7$. The core line-averaged electron density at $R=1.05 \mathrm{~m}$ is around $1.5 \times 10^{19} \mathrm{~m}^{-3}$. The plasma rotation is observed to be in the electron diamagnetic drift direction.

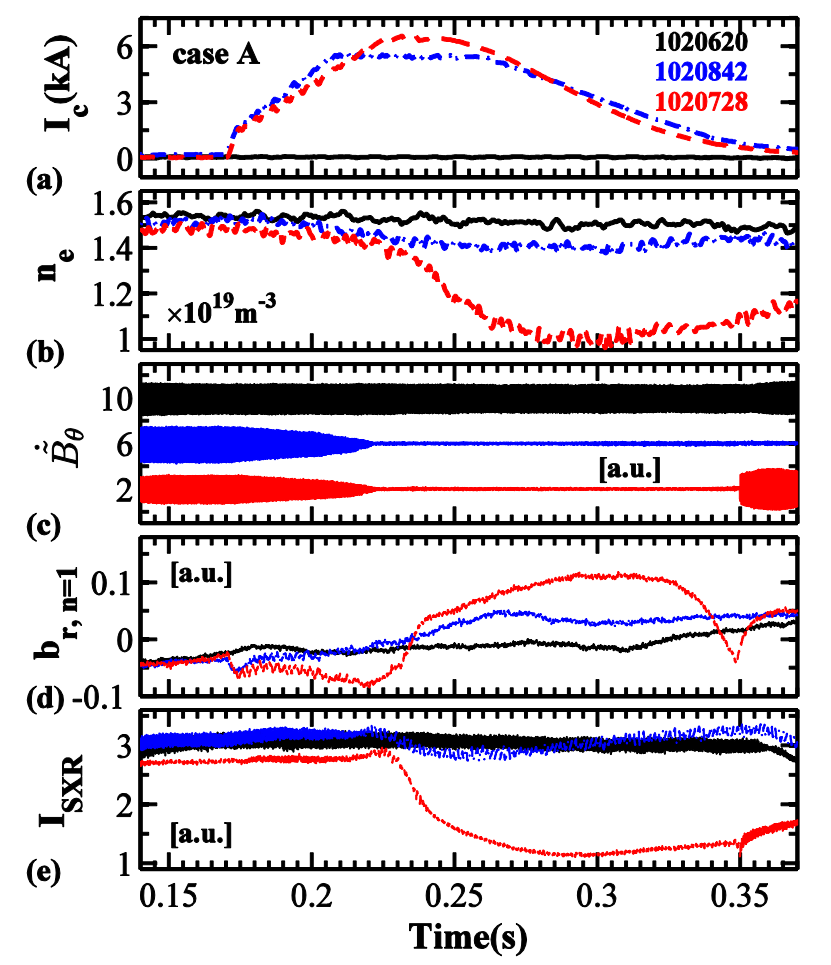

Figure 3. Experimental results with Case A spectrum. Time evolution of ( $a$ ) SRMP coils current $I_{c},(b)$ core line-averaged electron density $n_{\mathrm{e}}$ at $R=1.05 \mathrm{~m},(c)$ Mirnov signal $\mathrm{d} B_{\theta} / \mathrm{d} t$ (in arbitrary units), (d) locked mode detector signal $b_{r, \mathrm{n}=1}$ (in arbitrary units) and (e) intensity of soft X-ray emission intensity $I_{\mathrm{SXR}}$ (in arbitrary units) for shots 1020620 (black solid curve, $I_{c}=0$ ), 1020842 (blue dot-dashed curve, $I_{c}=5.5 \mathrm{kA}$ at the flattop) and 1020728 (red dashed curve, $I_{c}$ $=6.5 \mathrm{kA})$.

\section{Case A:}

Figure 3 shows the effect of SRMP amplitude in Ohmic discharges with the spectrum in Case A (figure 2(a)) and an existing 2/1 mode. The SRMP coil current $\left(I_{c}\right)$ is applied at $0.17 \mathrm{~s}$, then ramps up to $5.5 \mathrm{kA}$ in $0.04 \mathrm{~s}$ for shot 1020842 and to $6.5 \mathrm{kA}$ in $0.055 \mathrm{~s}$ for shot 1020728 (figure 3(a)), while shot 1020620 is a reference discharge without applying SRMP. During the application of a weaker SRMP in shot 1020842, the core line-averaged electron density at $R=1.05 \mathrm{~m}$ decreases from $1.51 \times 10^{19} \mathrm{~m}^{-3}$ to $1.39 \times 10^{19} \mathrm{~m}^{-3}$ as shown by the dot-dashed curve in figure $3(b)$. The relative change of the electron density, $\Delta n_{\mathrm{e}} / n_{\mathrm{e} 0}=\left(n_{\mathrm{e}}-n_{\mathrm{e} 0}\right) / n_{\mathrm{e} 0} \approx-8 \%$, where $n_{\mathrm{e} 0}$ is the line averaged density before application of SRMP, and $n_{\mathrm{e}}$ is the lowest one during application of SRMP. The Mirnov signal, $\mathrm{d} B_{\theta} / \mathrm{d} t$, is suppressed into noise level by applied RMPs (figure 3(c)), and there is no obvious change in the locked mode detector signal $b_{r}$ (figure $3(d)$ ), indicating the $2 / 1 \mathrm{TM}$ is stabilized. Although the electron density is slightly decreased, the soft X-ray emission intensity $I_{\mathrm{SXR}}$ increases (figure 3(e)). When a larger amplitude of SRMP is applied for shot 1020728 with $I_{c}=6.5 \mathrm{kA}$ at the flattop, the line averaged density decreases from $1.5 \times 10^{19} \mathrm{~m}^{-3}$ to $1 \times 10^{19} \mathrm{~m}^{-3}$, with $\Delta n_{\mathrm{e}} / n_{\mathrm{e} 0} \approx-33 \%$ as shown by the dashed curve in figure $3(b)$. Figure $3(c)$ shows that in this case the $2 / 1 \mathrm{TM}$ is first stabilized, but a locked mode is triggered later as indicated by a fast increase in $b_{r}$ (figure $3(d)$ ). Meanwhile a substantial decrease in $I_{\mathrm{SXR}}$ is observed as expected (figure $3(e)$ ). When $I_{c}$ ramps down to $0.62 \mathrm{kA}$ at $0.35 \mathrm{~s}$, the $2 / 1 \mathrm{TM}$ unlocks and spins up again as indicated by the reappearance of $\mathrm{d} B_{\theta} / \mathrm{d} t$ signal, and the plasma density begins to recover.

Corresponding to figure 3 , figure 4 shows the time evolution of the $m / n=2 / 1$ mode frequency for shots (a) 1020620, (b) 1020842 and (c) 1020728. During the application of SRMP, the mode frequency decreases from its initial value $f_{0}=6 \mathrm{kHz}$ to $4 \mathrm{kHz}$ for shot 1020842 and to $4.5 \mathrm{kHz}$ for 
1020728 before the mode disappears.

\section{Case B:}

For the spectrum in Case A utilized in figure 3, there are both the $m / n=2 / 1$ and 3/1 resonant components. For comparison, the experimental results (shot 1020634) with the spectrum in Case B, dominated only by the $m / n=3 / 1$ component (see figure 2(b)), is shown in figure 5. The SRMP coil current is ramped up to $7 \mathrm{kA}$ at the flattop (figure 5(a)). In this case, however, no change in the electron density has been observed as show in figure $5(b)$. When the time evolution of mode frequency and amplitude are examined, no changes have been observed. The reference results without applying RMPs (shot 1020620) are also shown in figure 5.

From figures 3 and 5, one finds that only the $m / n=2 / 1$ resonant component of applied SRMP has a significant effect on the particle transport as well as on the mode amplitude for discharges with an existing $2 / 1 \mathrm{TM}$, indicating that the decrease in the electron density is not due to the change in the wall condition (particle source) caused by RMPs, since otherwise there should also be a electron density decrease when applying the spectrum in Case B. The same gas puffing rate is applied in these discharges.

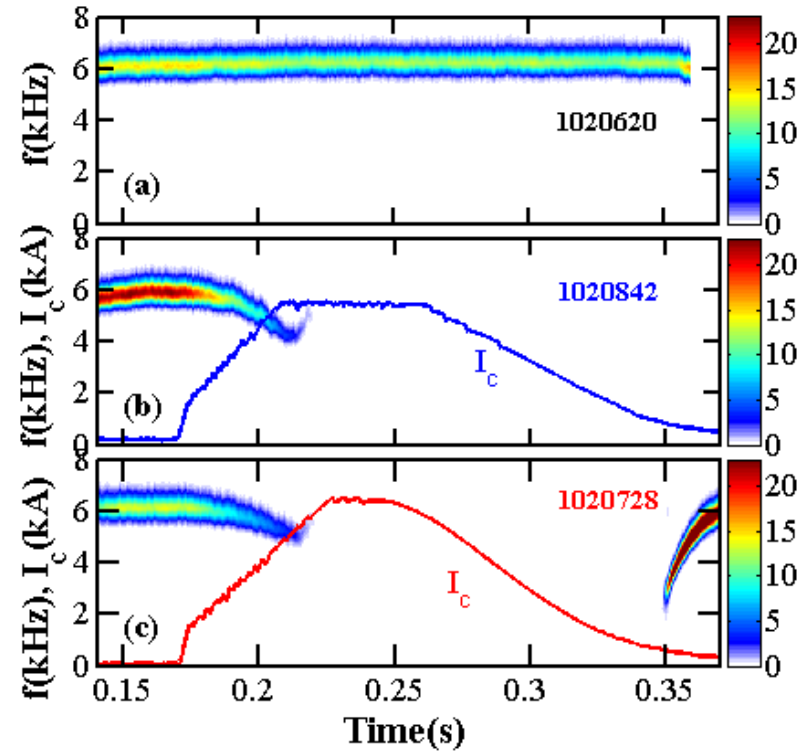

Figure 4. Corresponding to figure 3 , the wavelet power spectrum of Mirnov signal for (a) shot 1020620, (b) shot 1020842 and (c) shot 1020728. The mode frequency is $6 \mathrm{kHz}$ before applying SRMP, $\Delta f=f-f_{0}=$ $-2 \mathrm{kHz}$ for 1020842 , and $\Delta f=f-f_{0}=-1.5 \mathrm{kHz}$ for 1020728. Here the time trace of $I_{c}$ is superposed in $(b)$ and $(c)$.

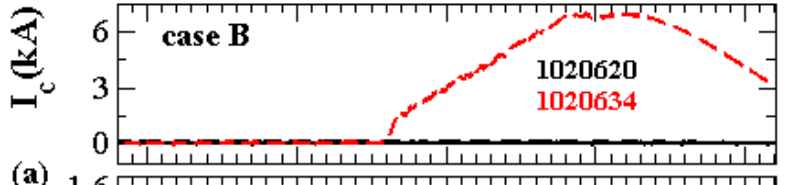

(a) $1.6 \mathrm{E}$.11

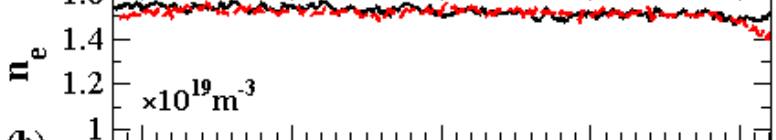

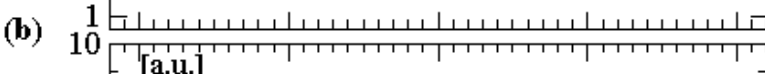

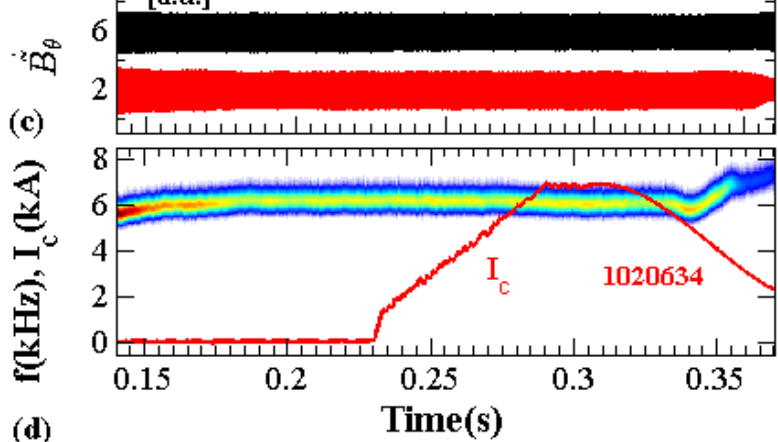

(d)

Figure 5. Experimental results with Case B spectrum. Time evolution of (a) SRMP coils current $I_{c},(b)$ core line-averaged electron density $n_{\mathrm{e}}$, (c) Mirnov signal $\mathrm{d} B_{\theta} / \mathrm{d} t$ (in arbitrary units), ( $d$ ) mode frequency for shots 1020620 (black solid curve) and 1020634 (red dashed curve). Here, $I_{c}$ equals $7 \mathrm{kA}$ at the flattop for shot 1020634 , the color bar of $(d)$ is the same as that of figure 4.

The relative change in the core plasma density, $\Delta n_{\mathrm{e}} / n_{\mathrm{e} 0}$, is shown as a function of $b_{2,1}^{1}(r=a)$ in figure 6, where $b^{1}{ }_{2,1}(r=a)$ is the $2 / 1$ component amplitude of SRMP at the plasma edge for different $I_{\mathrm{c}}$ and spectra. The plasma edge safety factor is $q_{a}=3.5$, and the initial $2 / 1$ mode frequency is $f_{0}=6$ $\mathrm{kHz}$ in these experiments. It is seen from figure 6 that there are two regimes: (1) For 2.5 Gauss $\leq$ 
$b^{1}{ }_{2,1}(r=a) \leq 4$ Gauss, there is a modest decrease in the electron density with $\Delta n_{\mathrm{e}} / n_{\mathrm{e} 0}$ ranges from $-3 \%$ to nearly $-10 \%$. The solid curve shows the relation $\Delta n_{\mathrm{e}} / n_{\mathrm{e} 0} \propto\left[b_{2,1}^{1}(r=a)\right]^{2}$ based on the quasi-linear prediction [27]. In this regime the mode stabilization by RMPs is also found. (2) For $b^{1}{ }_{2,1}(r=a)>4$ Gauss, there is a much larger decrease in the electron density with $\Delta n_{\mathrm{e}} / n_{\mathrm{e} 0} \sim-30 \%$, corresponding to large locked islands. It should be pointed out that the $2 / 1$ component of intrinsic error field is measured to be 0.31 Gauss [29], being much smaller than $b_{2,1}^{1}(a)$ shown in figure 6 , hence it is reasonable to neglect the intrinsic error field.

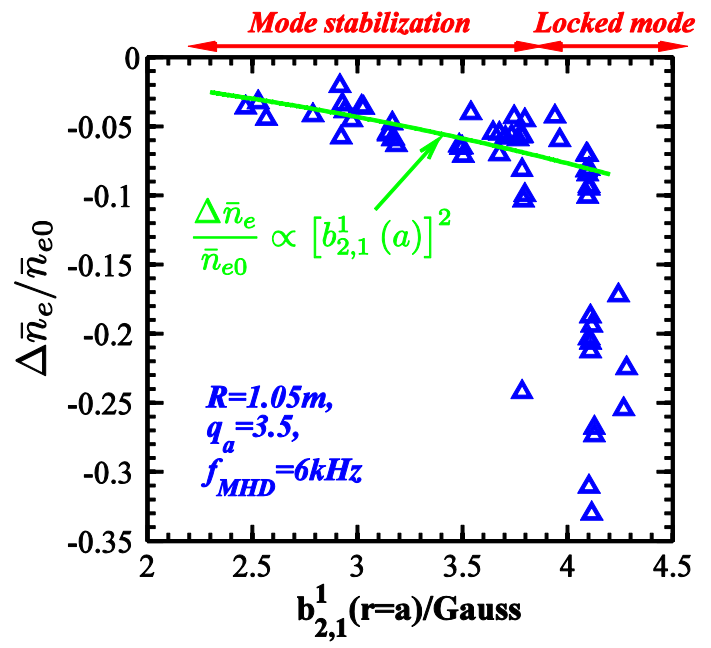

Figure 6. Relative change in core electron density $\Delta n_{\mathrm{e}} / n_{\mathrm{e} 0}$ versus the 2/1 component amplitude of perturbed radial magnetic field at plasma edge, $b^{1}{ }_{2,1}(r=a)$. For 2.5 Gauss $\leq$ $b^{1}{ }_{2,1} \leq 4$ Gauss, the $2 / 1 \mathrm{TM}$ is (partial) stabilized, and $3 \% \leq$ $\left|\Delta n_{\mathrm{e}} / n_{\mathrm{e} 0}\right|<10 \%$. For $b_{2,1}^{1}>4$ Gauss, locked modes are triggered, leading to $\left|\Delta n_{\mathrm{e}} / n_{\mathrm{e} 0}\right| \geq 20 \%$.

\section{Case C:}

In the experiments with applied DRMP [30], the electron cyclotron emission (ECE) radiometer was available for measuring the electron temperature $T_{\mathrm{e}}$ [37]. Since the ECE signals have not been calibrated, they only give the relative change in $T_{\mathrm{e}}$. DRMP is applied in shot 1027614 with an existing $2 / 1 \mathrm{TM}$, as shown in figure 7 . The plasma current is $I_{\mathrm{p}}=160 \mathrm{kA}$, the toroidal magnetic field $B_{\mathrm{t}}=1.8 \mathrm{~T}$, minor radius $a=0.265 \mathrm{~m}$, and $q_{a}=3.8$. The line averaged electron density is $1.6 \times 10^{19} \mathrm{~m}^{-3}$, and $I_{c}$ equals $5 \mathrm{kA}$ at the flattop. The initial $2 / 1$ mode frequency $f_{0}=7 \mathrm{kHz}$ before applying DRMP. In the time interval $0.26 \mathrm{~s}<t<0.36 \mathrm{~s}$, the $2 / 1 \mathrm{TM}$ is stabilized by DRMP as seen from figures $7(d)$ and $7(e)$, and wavelet analysis of the Mirnov signal shows that $\Delta f=-1.5 \mathrm{kHz}$. The electron density slightly decreases from $1.6 \times 10^{19} \mathrm{~m}^{-3}$ to $1.4 \times 10^{19} \mathrm{~m}^{-3}$ with $\Delta n_{\mathrm{e}} / n_{\mathrm{e} 0} \sim-12 \%$ (figure $7(b)$ ). However, an increase in electron temperature depicted by the ECE signal is observed (figure $7(c)$ ), which contributes to the increased $I_{\text {SXR }}$ (figure $7(f)$ ). The increase in the ECE signal can be divided into two phases in this time interval, a fast increase during the suppression of $2 / 1 \mathrm{TM}(0.26 \mathrm{~s}<t<0.28 \mathrm{~s})$ and a slow increase when $2 / 1 \mathrm{TM}$ is completely stabilized $(0.28 \mathrm{~s}<t<0.36 \mathrm{~s})$, which is marked by arrows in figure $7(c)$. The electron density is continuously decreased until $t=0.36 \mathrm{~s}$. After that time a locked mode is induced, as indicated by a sharp increase in $b_{\mathrm{r}}$ shown in figure $7(e)$. During the locked mode phase, substantial decreases are observed in $n_{\mathrm{e}}\left(\Delta n_{\mathrm{e}} / n_{\mathrm{e} 0} \sim-30 \%\right)$, ECE signal and $I_{\mathrm{SXR}}$. When $I_{c}$ ramps down later, the $2 / 1$ mode unlocks, and all the quantities (i.e. $n_{\mathrm{e}}$, ECE signal and $I_{\mathrm{SXR}}$ ) begin to recover. The results shown in figure 7 with the DRMP spectrum are similar to that shown in figure 3 , indicating again that only the $2 / 1$ resonant component of RMPs is important. 

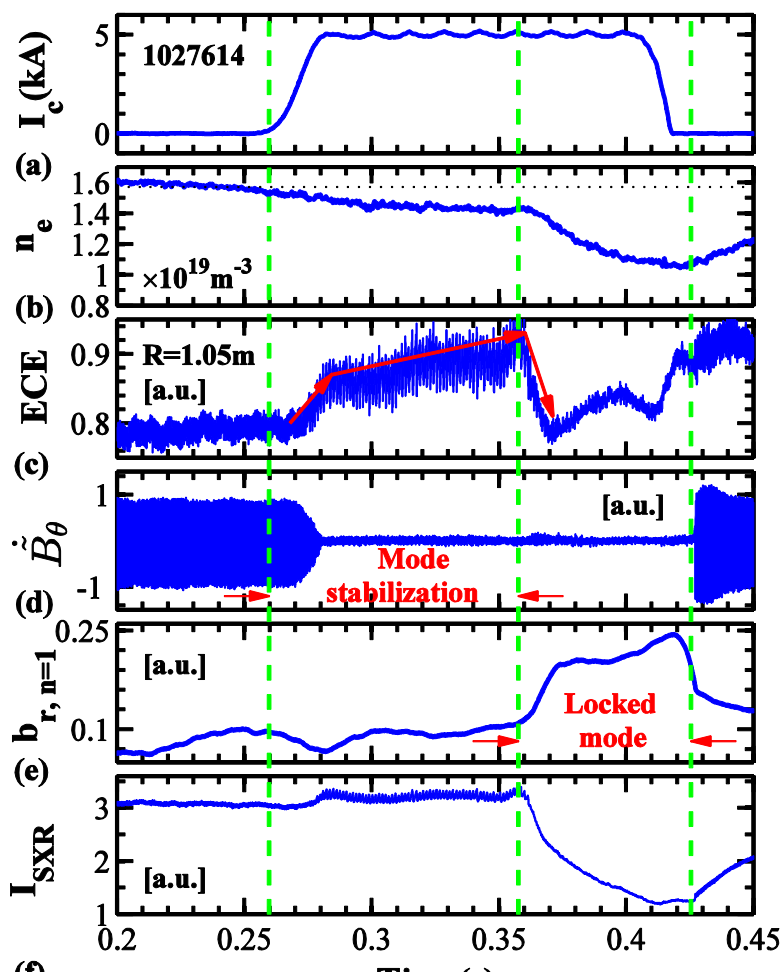

(f)

Time(s)

Figure 7. Experimental results with Case $\mathrm{C}$ spectrum. Time evolution of $(a)$ DRMP coils current $I_{c},(b)$ line-averaged electron density $n_{\mathrm{e}}$, (c) ECE signal (in arbitrary units), $(d) \mathrm{d} B_{\underline{\theta}} / \mathrm{d} t$ signal (in arbitrary units), $(e)$ locked mode detector signal $b_{\mathrm{r}}$ (in arbitrary units) and $(f)$ intensity of soft X-ray emission $I_{\text {SXR }}$ (in arbitrary units) for shots1027614. The mode is first stabilized by RMPs, but a locked mode is triggered later.

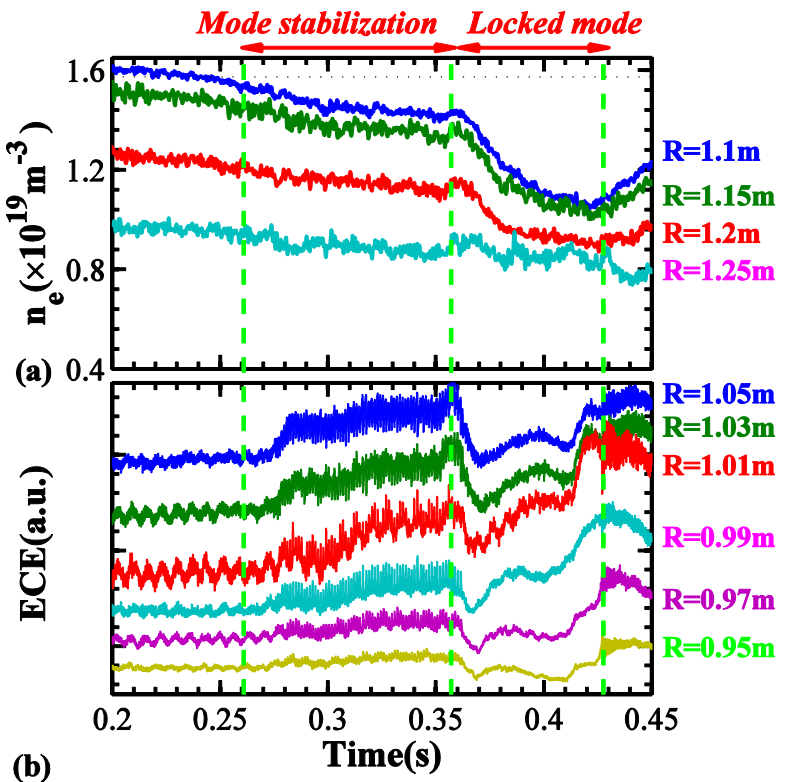

Figure 8. Corresponding to figure 7, time evolution of (a) electron density $n_{\mathrm{e}}$ and (b) ECE signals (in arbitrary units) at different radial locations. The $q=2$ surface is around $R=1.25 \mathrm{~m}$. During the time interval $0.26 \mathrm{~s}<t<0.36 \mathrm{~s}$, the $2 / 1 \mathrm{TM}$ is stabilized, accompanied by a decrease in $n_{\mathrm{e}}$ but an increase in the ECE signals. When locked mode is triggered later, both $n_{\mathrm{e}}$ and the ECE signals decrease sharply.

Corresponding to figure 7, the time evolution of $n_{\mathrm{e}}$ and the ECE signals (in arbitrary units) at different radial locations is shown in figure $8(a)$ and $8(b)$, respectively. The value of $R$ gives the major radius at which the line averaged values are measured along the vertical direction. The $q=2$ surface is estimated to be around $r=0.18 \mathrm{~m}$ (or $R=1.05 \mathrm{~m} \pm 0.18 \mathrm{~m}$ ). During the mode stabilization phase, the electron density decreases significantly for $R \leq 1.2 \mathrm{~m}$, which is located between the magnetic axis and the $q=2$ surface. An increase in the ECE signals is observed from all the channels with $0.95 \mathrm{~m} \leq R \leq 1.05 \mathrm{~m}$ on the high field side, which is also located between the magnetic axis and the $q=2$ surface. This again indicates that the changes of $n_{\mathrm{e}}$ and the ECE signals are not due to the variation in the wall condition, otherwise more significant changes in the edge region should be observed.

In figure 9, the effect of DRMP on originally tearing stable plasmas is shown. For these two shots, the plasma current is $I_{\mathrm{p}}=140 \mathrm{kA}$, toroidal magnetic field $B_{\mathrm{t}}=1.64 \mathrm{~T}$, minor radius $a=0.265 \mathrm{~m}$, and $q_{a}=3.92$. Before the application of DRMP, the line averaged electron density is $1.4 \times 10^{19} \mathrm{~m}^{-3}$, and no obvious oscillations are observed in the $\mathrm{d} B_{\theta} / \mathrm{d} t$ signal. For shot 1027453 with $I_{c}=4.5 \mathrm{kA}$, the applied DRMP causes $\Delta n_{\mathrm{e}} / n_{\mathrm{e} 0}$ to be nearly $-15 \%$ without triggering a locked mode, but no change in ECE, is observed (figure $9(c)$ ), which is different from the case as shown in figure 7 . For shot 1027454 with $I_{c}=5 \mathrm{kA}$, mode penetration occurs, and a substantial decrease in electron density and 
temperature is observed with $\Delta n_{\mathrm{e}} / n_{\mathrm{e} 0} \sim-30 \%$, similar to the locked mode phase shown in figure 7. Analysis based on $\mathrm{d} B_{\theta} / \mathrm{d} t$ in figure $9(d)$ shows that a $2 / 1$ locked mode is triggered by RMPs.

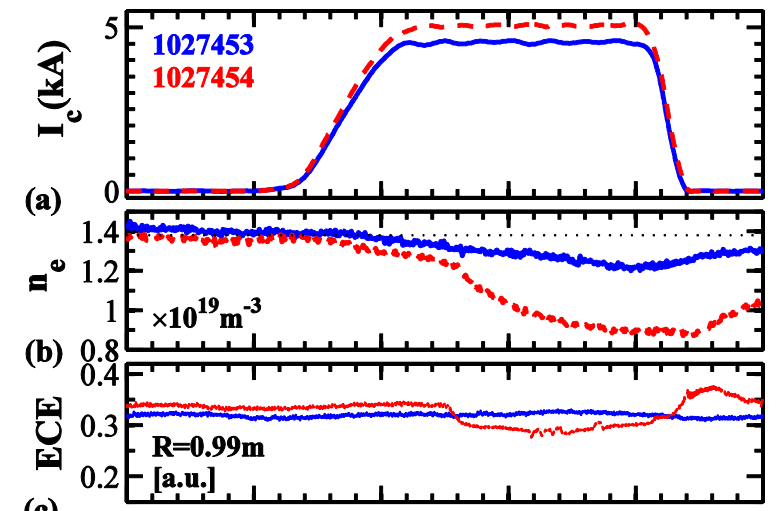

(c)
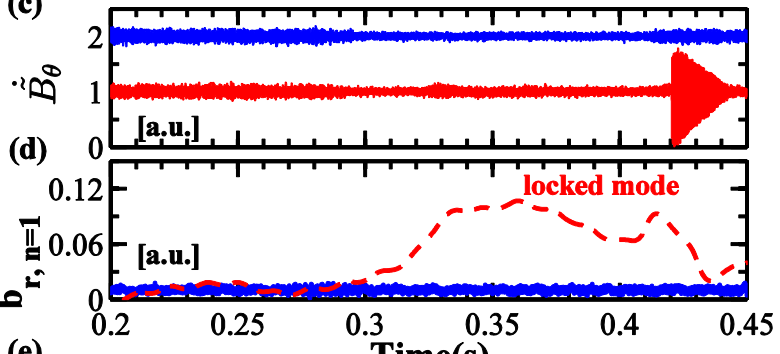

(e)

Time(s)
Figure 9. DRMP effect on initially tearing stable plasmas. Time evolution of (a) DRMP coils current $I_{c}$, (b) line-averaged electron density $n_{\mathrm{e}},(c)$ ECE signal (in arbitrary units), (d) Mirnov signal $\mathrm{d} B_{\theta} / \mathrm{d} t$ (in arbitrary units) and (e) locked mode detector signal $b_{r}$ (in arbitrary units) for shots $1027453\left(I_{c}=4.5 \mathrm{kA}\right.$, solid cure) and $1027454\left(I_{c}=5 \mathrm{kkA}\right.$, dashed cure). Locked mode is triggered for 1027454 .

When the applied RMP is not strong enough to cause a locked mode or mode penetration, the decrease in mode frequency is very limited [16]. Taking shot 1027614 as an example, $\left|\Delta f / f_{0}\right| \sim 20 \%$ is observed.

Table 1. Summary of RMP effect on density change and MHD for 3 cases

\begin{tabular}{|c|c|c|c|c|}
\hline \multirow{2}{*}{$\begin{array}{c}\text { RMP } \\
\text { Spectrum }\end{array}$} & \multirow{2}{*}{ MHD } & \multicolumn{2}{|c|}{$\Delta n_{\mathrm{e}} / n_{\mathrm{e} 0}$} & \multirow{2}{*}{ Effect } \\
\hline & & $\begin{array}{c}R=1.05 \mathrm{~m} \\
(r / a=0)\end{array}$ & $\begin{array}{c}R=1.2 \mathrm{~m} \\
(r / a=0.57)\end{array}$ & \\
\hline \multirow{2}{*}{$\begin{array}{c}\text { Case A } \\
2 / 1,3 / 1\end{array}$} & \multirow{2}{*}{ 2/1 TM } & $-7.8 \%$ & $-4.2 \%$ & mode stabilization (i.e. 1020842) \\
\hline & & $-31.7 \%$ & $-19.5 \%$ & locked mode (i.e. 1020728) \\
\hline $\begin{array}{c}\text { Case } B \\
3 / 1 \\
\end{array}$ & 2/1 TM & 0 & 0 & no effect (i.e. 1020728) \\
\hline \multirow{3}{*}{$\begin{array}{c}\text { Case } C \\
2 / 1\end{array}$} & 2/1 TM & $-30 \%$ & $-23 \%$ & mode stabilization (i.e. 1027614) \\
\hline & \multirow{2}{*}{ W/O TM } & $-15 \%$ & $-9.2 \%$ & W/O penetration (i.e. 1027453 ) \\
\hline & & $-34.6 \%$ & $-22.0 \%$ & Penetration (i.e. 1027454) \\
\hline
\end{tabular}

In summary, by using RMPs with different spectra and amplitudes, two types of electron density decrease caused by applied RMPs have been observed in J-TEXT discharges as shown in table 1: (a) If no locked mode is triggered, a relatively small decrease in the electron density, $\Delta n_{\mathrm{e}} / n_{\mathrm{e}} 0$ $\sim-10 \%$, is found, but the electron temperature increases accompanying the mode stabilization. The changes of $n_{\mathrm{e}}$ and the ECE signal are in the core region inside the $q=2$ surface, and the closer to the plasma core the stronger the change will be. (b) When a locked mode or mode penetration is triggered by sufficiently strong RMPs, there is a much larger decrease in the electron density 
$\left(\Delta n_{\mathrm{e}} / n_{\mathrm{e} 0} \sim-30 \%\right)$ as well as in the electron temperature. Only the $2 / 1$ component of RMPs has been found to affect the electron density as well as the mode amplitude.

\section{Numerical results}

\subsection{Theoretical model}

In order to understand and compare with the experimental results shown in section 2, the two-fluid equations are used in calculations under the large aspect-ratio approximations. The magnetic field is defined as $\boldsymbol{B}=B_{0 t} \boldsymbol{e}_{\mathrm{t}}-(\mathrm{kr} / \mathrm{m}) B_{0 t} \boldsymbol{e}_{\theta}+\nabla \psi \times \boldsymbol{e}_{\mathrm{t}}$, where $\psi$ is the helical flux function, $m / r$ and $k=n / R$ are the wave vectors in the $\boldsymbol{e}_{\theta}$ (poloidal) and $\boldsymbol{e}_{\mathrm{t}}$ (toroidal) direction, respectively, $R$ is the major radius and the subscript 0 denotes an equilibrium quantity. The ion velocity $\boldsymbol{v}=v_{\|} \boldsymbol{e}_{\|}+\boldsymbol{v}_{\perp}$, where $v_{\|}$ and $v_{\perp}=\nabla \phi \times \boldsymbol{e}_{\mathrm{t}}$ are the parallel (to the magnetic field) and the perpendicular velocity, respectively. Here, $\phi$ is the stream function.

The two-fluid equations utilized here include the mass conservation equation, the generalized Ohm's law and the equation of motion in the perpendicular (after taking $\boldsymbol{e}_{\mathrm{t}} \cdot \nabla \times$ ) and parallel (to magnetic field) directions. Normalizing the length to the minor radius $a$, the time $t$ to the resistive time $\tau_{\mathrm{R}}=a^{2} \mu_{0} / \eta$, the helical flux $\psi$ to $a B_{0 \mathrm{t}}, v$ to $a / \tau_{\mathrm{R}}$ and the electron density $n_{\mathrm{e}}$ to its value at the magnetic axis, these equations becomes $[38,39]$

$$
\begin{aligned}
& \frac{\mathrm{d} n_{\mathrm{e}}}{\mathrm{d} t}=d_{1} \nabla_{\|} j-\nabla_{\|}\left(n_{\mathrm{e}} v_{\|}\right)+\nabla_{\perp}\left(D_{\perp} \nabla_{\perp} n_{\mathrm{e}}\right)+S_{\mathrm{n}}, \\
& \frac{\mathrm{d} \psi}{\mathrm{d} t}=E_{0}-\eta j-\Omega \nabla_{\|} n_{\mathrm{e}}, \\
& \frac{\mathrm{d} U}{\mathrm{~d} t}=-S^{2} \nabla_{\|} j+\mu \nabla_{\perp}^{2} U+S_{\mathrm{m}}, \\
& \frac{\mathrm{d} v_{\|}}{\mathrm{d} t}=-c_{\mathrm{s}}^{2} \nabla_{\|} P / n_{\mathrm{e}}+\mu \nabla_{\perp}^{2} v_{\|},
\end{aligned}
$$

where $\mathrm{d} / \mathrm{d} t=\partial / \partial t+v_{\perp} \cdot \nabla_{\perp}, j$ is the parallel plasma current density, $\eta$ is the normalized resistivity, $E_{0}$ the equilibrium electric field, $U=-\nabla_{\perp}^{2} \phi$ the plasma vorticity, $S_{\mathrm{n}}$ the particle source and $S_{\mathrm{m}}$ the poloidal momentum source leading to an equilibrium poloidal plasma rotation. $d_{1}=\omega_{\mathrm{ce}} / v_{\mathrm{e}}, \omega_{\mathrm{ce}}$ and $v_{\mathrm{e}}$ are the electron cyclotron and the collisional frequency, $\Omega=\beta d_{1}, \beta=4 \pi P_{\mathrm{e}} / B_{0 \mathrm{t}}{ }^{2}, P=P_{\mathrm{e}}+P_{\mathrm{i}}, P_{\mathrm{e}}$ $\left(P_{\mathrm{i}}\right)$ is the electron (ion) pressure, $S=\tau_{\mathrm{R}} / \tau_{\mathrm{A}}$, where $\tau_{\mathrm{A}}=a / V_{\mathrm{A}}$ is the toroidal Alfvén time. $c_{\mathrm{s}}, \mu$ and $D_{\perp}$ are the normalized ion sound velocity, plasma viscosity and perpendicular particle diffusivity.

Based on equations (1)-(4), the quasi-linear analysis shows that the change in local plasma density gradient by a static RMP is given by [27]

$$
r_{\mathrm{s}}\left(\Delta n_{\mathrm{e}}\right)^{\prime} / n_{\mathrm{e}}=d_{1}\left(\omega_{* \mathrm{e} 0} r_{\mathrm{s}}^{2} / D_{\perp}\right)\left(1-\omega_{0}\right)\left|b_{1 \mathrm{r}} / B_{\mathrm{Ot}}\right|^{2} / m
$$

at the resonant surface $r=r_{\mathrm{s}}$ in steady state, where $\Delta n_{\mathrm{e}}=\left(n_{\mathrm{e}, 0 / 0}-n_{\mathrm{e} 0}\right), n_{\mathrm{e}, 0 / 0}$ is the $m / n=0 / 0$ component of the electron density. $\omega_{0}=-\omega_{\mathrm{E} 0} / \omega *_{\mathrm{e} 0}$ is the ratio between the equilibrium plasma rotation angular frequency $\omega_{\mathrm{E} 0}$ and the electron diamagnetic drift angular frequency $\omega * \mathrm{e} 0$.

Equations (1)-(4) are solved using the initial value code TM1 [27]. The calculations are performed for a single helicity of RMP with $m / n=2 / 1$, being taken into account by the boundary condition

$$
\left.\psi_{2 / 1}\right|_{r=a}=\psi_{a} a B_{0 \mathrm{t}} \cos (m \theta+n \varphi)
$$


where $\psi_{a}$ describes the normalized $m / n=2 / 1$ helical magnetic flux amplitude at $r=a$. The radial magnetic field perturbations at $r=a$ is given by $b^{1}{ }_{2,1}(r=a)=-m \psi_{a} B_{0 \mathrm{t}} \sin (m \theta+n \varphi)$.

\subsection{Numerical results}

The input parameters are based on experimental parameters as mentioned in section 2. A monotonic $q$ profile is used with the $q=2$ surface located at $r_{\mathrm{s}}=0.7 a$ [31]. The $\mathrm{m} / \mathrm{n}=2 / 1$ tearing mode is unstable without applying RMPs. The toroidal magnetic field is $B_{0 \mathrm{t}}=1.75 \mathrm{~T}$, the plasma minor and major radius are $a=0.265 \mathrm{~m}$ and $R=1.05 \mathrm{~m}$. At the $q=2$ surface, $n_{\mathrm{e}}=1 \times 10^{19} \mathrm{~m}^{-3}$ and $T_{\mathrm{e}}=$ $350 \mathrm{eV}$ are utilized, resulting in the resistive time $\tau_{\mathrm{R}}=0.2 \mathrm{~s}$. The following parameters, $S=1 \times 10^{7}, d_{1}$ $=2.5 \times 10^{6}, \Omega=4.6 \times 10^{2}, c_{\mathrm{s}}=1.6 \times 10^{5}\left(a / \tau_{\mathrm{R}}\right)$, and the plasma viscosity and perpendicular particle diffusivity at the anomalous transport level with $\mu=5 D_{\perp}=0.5 \mathrm{~m}^{2} \mathrm{~s}^{-1}$ are used except mentioned elsewhere. The plasma rotation is taken to have a frequency $f_{\mathrm{E} 0}=5 \mathrm{kHz}$ at the $q=2$ surface and rotate in the electron diamagnetic drift direction. The electron diamagnetic drift frequency is about $f_{* \mathrm{e} 0} \sim 1 \mathrm{kHz}$. Hence the frequency of the $2 / 1 \mathrm{TM}$ is $f_{2 / 1}=f_{\mathrm{E} 0}+f_{*_{\mathrm{e}} 0}=6 \mathrm{kHz}$, with $\omega_{0}=-\omega_{\mathrm{E} 0} / \omega *_{\mathrm{e} 0}=$ $-f_{\mathrm{E} 0} / f_{\mathrm{e} 0}=-5$. Based on the above parameters, the obtained numerical results are presented in figures $10-14$.

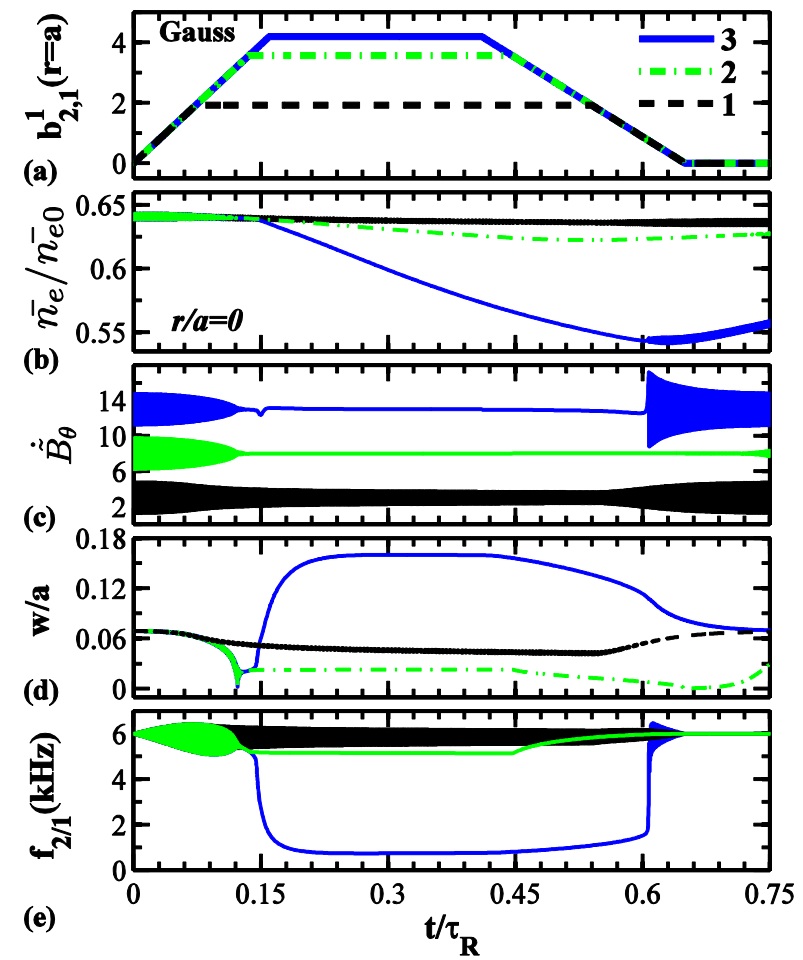

Figure 10. Time evolution of $(a)$ applied $2 / 1$ RMP $b^{1}{ }_{2,1}$ in unit of Gauss, (b) normalized line-averaged electron density $n_{\mathrm{e}} / n_{\mathrm{e} 0},(c)$ calculated $\mathrm{d} B_{\theta} / \mathrm{d} t,(d)$ normalized island width $w / a$ and $(e) 2 / 1$ mode frequency $f_{2 / 1}$ for three values of $b_{2,1}^{1}$ are shown. Here, $b_{2,1}^{1}(a)=1.8$ Gauss for case 1 (black dashed curve), 3.6 Gauss for case 2 (green dot-dashed curve) and 4.2 Gauss for case 3 (blue solid curve).
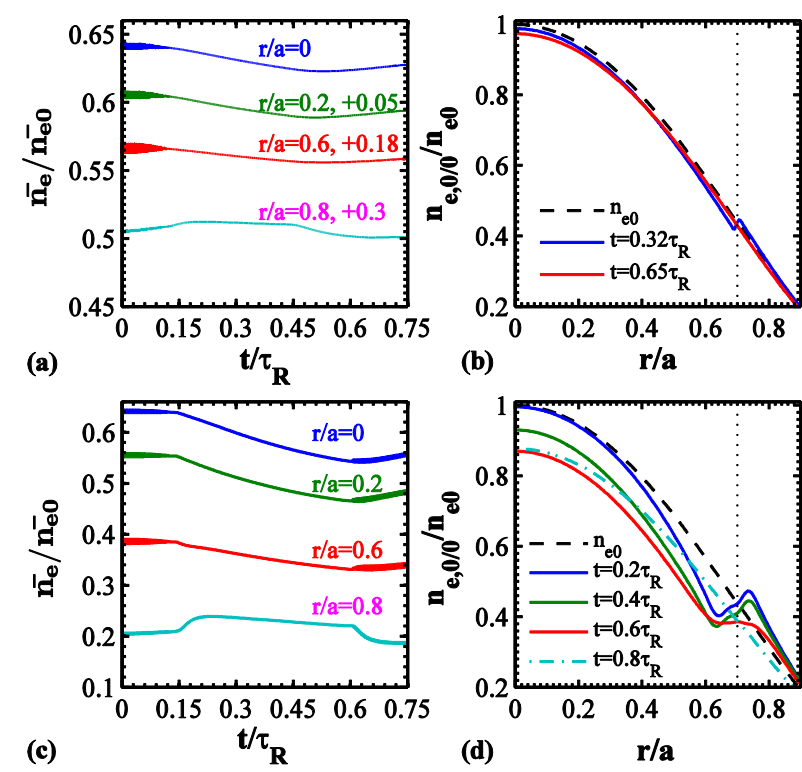

(b)

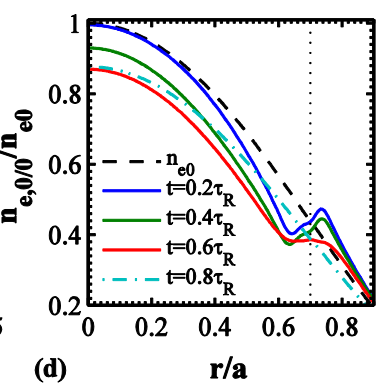

Figure 11. Corresponding to figure 10, (a) time evolution of $n_{\mathrm{e}} / n_{\mathrm{e} 0}$ at 4 different radial locations and $(b)$ radial profile of $n_{\mathrm{e}, 0 / 0} / n_{\mathrm{e} 0}$ at different time for case 2. Time evolution of $n_{\mathrm{e}} / n_{\mathrm{e} 0}$ and radial profile of $n_{\mathrm{e}, 0 / 0} / n_{\mathrm{e} 0}$ for case 3 are shown in (c) and (d), respectively.

In figure 10, effects of the $m / n=2 / 1 \mathrm{RMP}$ on an existing $2 / 1 \mathrm{TM}$ and the particle transport are shown. Before the application of RMP, the 2/1 TM saturates at an island width $w=0.072 a$. 
Afterwards the 2/1 RMP is applied as shown in figure 10(a), with $b^{1}{ }_{2,1}(a)$ equals 1.8 Gauss for case 1, 3.6 Gauss for case 2 and 4.2 Gauss for case 3 at the flattop. For case 1 with a weak RMP, the 2/1 island width is slightly reduced as shown in figure $10(d)$, and the density $n_{\mathrm{e}} / n_{\mathrm{e} 0}$ (figure $10(b)$ ) and mode frequency $f_{2 / 1}$ (figure $10(e)$ ) change little. For case 2 with a stronger RMP, the island width is suppressed to a small value, and $\mathrm{d} B_{\theta} / \mathrm{d} t$ decreases to zero. In addition, slight decrease in electron density and mode frequency have been found with $\Delta n_{\mathrm{e}} / n_{\mathrm{e} 0} \sim-7 \%$ and $\Delta f_{2 / 1} / f_{0} \sim-20 \%$, which agrees with the mode stabilization case of shots 1020842 and 1027614 shown in figure 3 and figure 7. When the applied RMP is strong enough, a locked mode is triggered after mode stabilization as shown for case 3. The saturated island width is $w=0.16 a$, resulting in a large decrease in electron density with $\Delta n_{\mathrm{e}} / n_{\mathrm{e} 0} \sim-25 \%$, and the mode frequency $f_{2 / 1}$ and calculated $\mathrm{d} B_{\theta} / \mathrm{d} t$ decrease to near zero. Case 3 is similar to experimental results with locked modes (shots 1020728 and 1027614).

Corresponding to the cases 2 and 3 in figure 10, the time evolution of line averaged electron density $n_{e}$ at different radial locations and radial profiles of $n_{e}$ are shown in figure 11. Figure 11(a) and $11(c)$ indicate that the RMP only causes a density decrease inside the $q=2$ surface $(r<0.7 a)$ for both the mode stabilization and the locked mode case, which is consistent with experimental results shown in section 2. Similar to the experimental observation (shot 1027614), a density increase outside the $q=2$ surface $(r>0.7 a)$ is also seen after a locked mode is triggered (figure 11(c)). The radial profiles of $n_{\mathrm{e}, 0 / 0}$ shown in figure $11(b)$ and $11(\mathrm{~d})$ indicate that the enhanced particle transport begins from a layer around the $q=2$ surface and then spreads over the region between the magnetic axis and the $q=2$ surface. At the time of $t=0.2 \tau_{\mathrm{R}}$ in figure $11(d)$, the locked mode is triggered, and the density changes in the opposite way on the two sides of the $q=2$ surface. The large magnetic island eventually flattens the density profile around $r_{\mathrm{s}}$ and results in a large decrease in the electron density. When the RMP is ramped down, the $2 / 1$ mode unlocks, and the density profile begin to recover as shown by the dot-dashed curve in figure $11(\mathrm{~d})$ at $t=0.8 \tau_{\mathrm{R}}$.
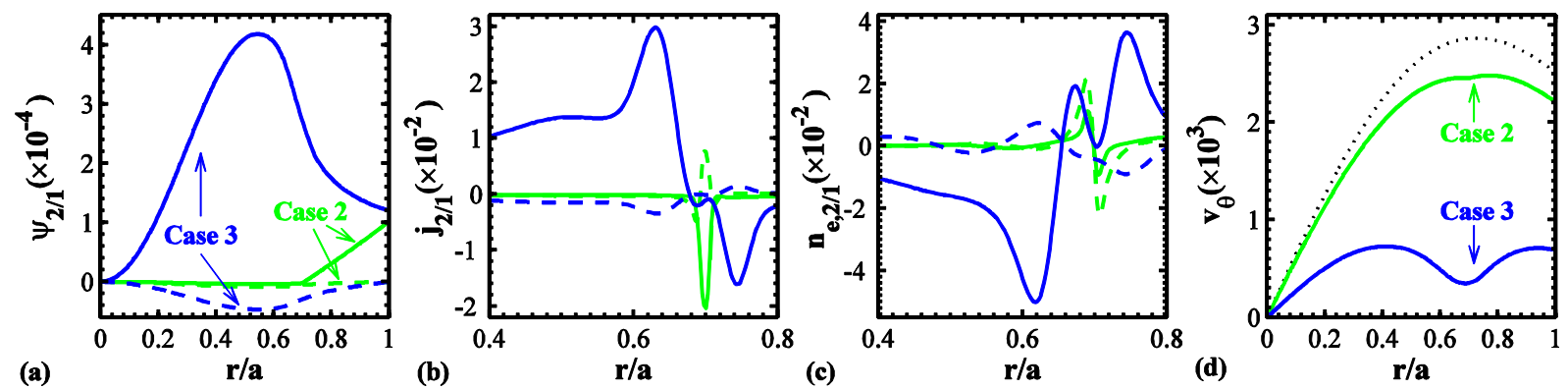

Figure 12. Corresponding to the time $t=0.32 \tau_{\mathrm{R}}$ of figure 10 , radial profiles of ( $a$ ) the $2 / 1$ component of perturbed helical flux $\psi_{2 / 1},(b) 2 / 1$ component of plasma current density $j_{2 / 1}$ and (c) 2/1 component of perturbed electron density $n_{\mathrm{e}, 2 / 1} / n_{\mathrm{e} 0}$ are shown for cases 2 (green curves) and 3 (blue curves). The solid (dashed) cures represent the real (imaginary) part. Figure 12(d) shows the radial profile of poloidal rotation velocity $v_{\theta}$ for cases 2 (green curve) and 3 (blue curve) at $t=0.32 \tau_{\mathrm{R}}$, and the dotted curve is the original equilibrium profile of $v_{\theta}$.

Corresponding to the time of $t=0.32 \tau_{\mathrm{R}}$ in figure 10, radial profiles of perturbed helical flux $\psi_{2 / 1}$, plasma current density $j_{2 / 1}$, electron density $n_{\mathrm{e}, 2 / 1} / n_{\mathrm{e} 0}$, and the plasma poloidal velocity $v_{\theta}$ for cases 2 (green curve) and 3 (blue curve) are shown in figure 12(a-d). For case 2, the applied RMP is shielded inside the $q=2$ surface by the local screening current localized at $r_{\mathrm{s}}$ as shown in figure 12(b). The perturbed electron density is also localized around $r_{\mathrm{s}}$. The braking effect contributed by RMP on the plasma rotation is weak, as shown in figure $12(d)$, while for case 3 the perturbed field is much larger, and the poloidal rotation $v_{\theta}$ is reduced to nearly zero at $r_{\mathrm{s}}$. 

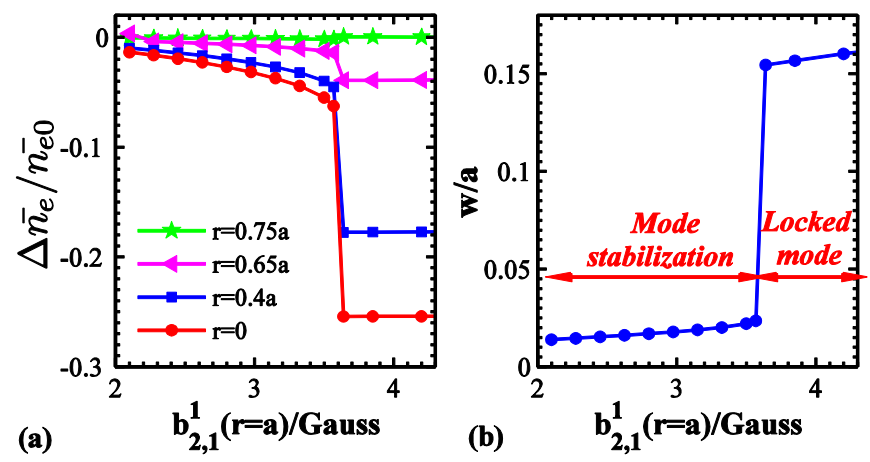

Figure 13. (a) Normalized relative changes in $\Delta n_{\mathrm{e}} / n_{\mathrm{e} 0}$ at different radial locations and (b) saturated island width $w / a$ versus the applied field $b_{2,1}^{1}(a)$. Both mode stabilization and locked mode regimes have been found, similar to experimental observations shown in figure $6 . \Delta n_{\mathrm{e}} / n_{\mathrm{e} 0} \sim-2 \%$ to $-7.5 \%(\sim-25 \%)$ at $r=0$ in the mode stabilization (locked mode) regime.

By scanning the amplitude of the applied field $b_{2,1}^{1}$ in calculations, the relative change in line -averaged electron density and saturated island width are shown as a function of $b^{1}{ }_{2,1}$ in figure 13(a) and $13(b)$, respectively. Both the mode stabilization and locked mode regimes have been numerically obtained. For 2 Gauss $<b_{2,1}^{1}<3.55$ Gauss, the $2 / 1 \mathrm{TM}$ is stabilized by RMPs, and the island width $w / a<0.024$. In this case the values of $\Delta n_{\mathrm{e}} / n_{\mathrm{e} 0}$ are between $-2 \%$ and $-7.5 \%$. For $b^{1}{ }_{2,1} \geq$ 3.55 Gauss, a $2 / 1$ locked mode is triggered, resulting in a large island with w/a $>0.16$, and the relative change of the electron density is larger than $-25 \%$. The results shown in figure 13 agree with the experimental ones shown in figure 6.
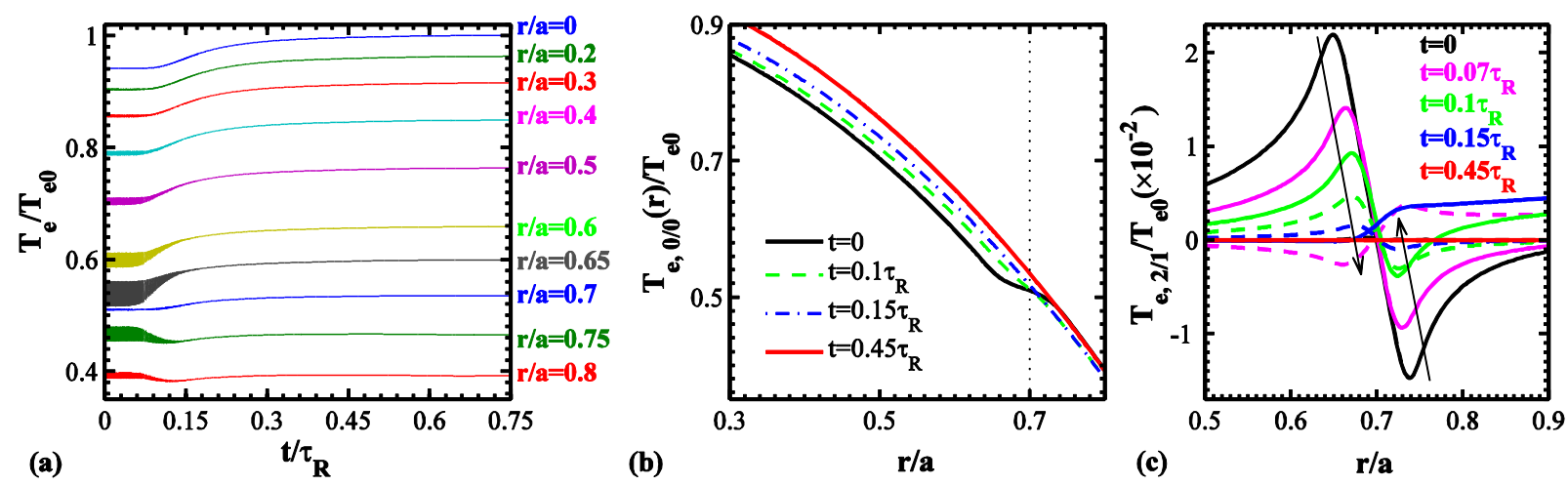

Figure 14. Corresponding to case 2 in figure 10, (a) time evolution of $T_{\mathrm{e}} / T_{\mathrm{e} 0}$ at 10 different radial positions, $(b)$ radial profile of $T_{\mathrm{e}, 0 / 0} / T_{\mathrm{e} 0}$ at different time and (c) corresponding radial profiles of $T_{\mathrm{e}, 2 / 1} / T_{\mathrm{e} 0}$ are shown. In (c) the solid (dashed) curves represent the real (imaginary) part.

In the experimental results shown in section 2, the increase in ECE is observed accompanying the mode stabilization. By using single-fluid equations taking into account the energy conservation equation [40], the numerical results are shown in figure 14. All the input parameters are the same as those used for case 2 in figure 10. Figure 14(a) shows the time evolution of $T_{\mathrm{e}}$ at different radial locations. During $0<t<0.15 \tau_{\mathrm{R}}$, the $2 / 1 \mathrm{TM}$ is suppressed, and $T_{\mathrm{e}}$ quickly increases for $r<r_{\mathrm{s}}$, but decreases for $r>r_{\mathrm{s}}$. After the 2/1 TM is completely stabilized for $t>0.15 \tau_{\mathrm{R}}, T_{\mathrm{e}}$ continues to slowly increase inside $r_{\mathrm{s}}$. The results shown in figure 14(a) agree with experimental ones shown in figure 7-8. In figure 14(b) radial profiles of $T_{\mathrm{e}}$ at different time are shown. Before the RMP is applied, the saturated 2/1 island flattens the local $T_{\mathrm{e}}$ profile around $r_{\mathrm{s}}$ as shown by the curve at the time $t=0$. During the application of RMP, the $2 / 1$ island width is decreased, resulting in the increase in $T_{\mathrm{e}}$ until 
the $2 / 1 \mathrm{TM}$ is stabilized. The radial profiles of the perturbed $T_{\mathrm{e}, 2 / 1}$ are shown in figure $14(c)$. The peaks in the $T_{\mathrm{e}, 2 / 1}$ profiles are at the island edge and move towards the $q=2$ surface, as indicated by the arrows.

\section{Discussion and summary}

By using both SRMP and DRMP, the resonant effects on particle transport have been studied in J-TEXT tokamak in the conditions of edge safety factor $q_{a} \sim 3.5-3.9$ with plasma rotating in the electron diamagnetic drift direction. It is found that for tearing-unstable plasmas, the response to applied RMP can be divided into two regimes - mode stabilization regime and locked mode regime. In the mode stabilization regime, the initially saturated $2 / 1 \mathrm{TM}$ is completely stabilized, resulting in slight electron density change with $\Delta n_{\mathrm{e}} / n_{\mathrm{e} 0} \sim-3 \%$ to $-10 \%$, and the value of $\Delta n_{\mathrm{e}} / n_{\mathrm{e} 0}$ is proportional to the square of the field amplitude. In addition, the plasma rotation reduction is less than $50 \%$, and an increase in the ECE signal is observed too. In the locked mode regime, both $n_{\mathrm{e}}$ and the ECE signal are significantly decreased due to the large island. For tearing stable plasmas, the results are similar to the tearing unstable case.

Based on the two-fluid equations, numerical modeling is carried out. Both mode stabilization and locked mode regimes have been obtained in calculations too. When the $2 / 1 \mathrm{TM}$ is stabilized, the perturbed field is shielded by the screening current at the $2 / 1$ resonant surface as shown by figure 12 , which causes a weak reduction in $n_{\mathrm{e}}$ and plasma rotation [17]. Besides, the reduced island width decreases the flatten effect on $T_{\mathrm{e}}$ contributed by magnetic island [41], resulting in increase in $T_{\mathrm{e}}$. When the field is strong enough, a locked mode is triggered. The substantial reduction in $n_{\mathrm{e}}$ and $T_{\mathrm{e}}$ is due to the large magnetic island.

In summary, the effect of RMPs on particle transport has been studied in J-TEXT ohmic plasmas by using both the SRMP and DRMP coils. The major results are as follows:

(1) For plasma with an initially saturated 2/1 TM, applied SRMP of moderate amplitude (2.5Gauss $\leq b_{2,1}^{1} \leq 4$ Gauss) leads to mode stabilization and a reduction in electron density with a relative amplitude $\left|\Delta n_{\mathrm{e}} / n_{\mathrm{e} 0}\right|<10 \%$, while the electron temperature increases. When the applied RMPs are sufficiently large (i.e. $b_{2,1}^{1}>4$ Gauss), a locked mode is triggered, resulting in a large decrease in $n_{\mathrm{e}}\left(\left|\Delta n_{\mathrm{e}} / n_{\mathrm{e} 0}\right| \geq 20 \%\right)$.

(2) In the mode stabilization regime, the relative change in $n_{\mathrm{e}}$ is proportional to the square of the RMP amplitude $-\Delta n_{\mathrm{e}} / n_{\mathrm{e} 0} \propto\left[b^{1}{ }_{2,1}(r=a)\right]^{2}$, being consistent with quasi-linear predictions [27]. In this regime the reduction in mode frequency is small $(|\Delta f / f| \sim 20 \%)$, and an increase in the electron temperature is observed.

(3) Utilizing DRMP, similar experimental results to those with SRMP are found.

(4) With experimental parameters as input, the numerical results agree with experimental ones.

\section{Acknowledgements}

We thank Professor K.W. Gentle for carefully reviewing and correcting the manuscript. This work is supported by the Ministry of Science and Technology (Contract No. 2011GB109001 and 2010GB107004) and National Natural Science Foundation (Contract No. 10990214 and 11275080) and the Chang-Jiang scholar project of the Ministry of Education, China.

\section{References}

[1] Evans T. E. et al 2004 Phys. Rev. Lett. 92235003 
[2] Liang Y. et al 2007 Phys. Rev. Lett. 98265004

[3] Kirk A. et al 2010 Nucl. Fusion 50034008

[4] Suttrop W. et al 2011 Phys. Rev. Lett. 106225004

[5] Jeon Y. M. et al 2012 Phys. Rev. Lett. 109035004

[6] Mordijck S. et al 2012 Phys. Plasmas 19056503

[7] Finken K. H. et al 2007 Phys. Rev. Lett. 98065001

[8] Schmitz O. et al 2012 Nucl. Fusion 52043005

[9] Hender T. C. et al 1992 Nucl. Fusion 322091

[10] Fitzpatrick R. 1993 Nucl. Fusion 331049

[11] Tokar M. Z. et al 2007 Phys. Rev. Lett. 98095001

[12] Schmitz O. et al 2009 Phys. Rev. Lett. 103165005

[13] Rozhansky V. et al 2010 Nucl. Fusion 50034005

[14] Schmitz O. et al 2009 J. Nucl. Mater. 390-91 330

[15] Liu Y. Q. et al 2011 Nucl. Fusion 51083002

[16] Yu Q., Gunter S. 2011 Nucl. Fusion 51073030

[17] Waelbroeck F. L. et al 2012 Nucl. Fusion 52074004

[18] Becoulet M. et al 2012 Nucl. Fusion 52054003

[19] Kaveeva E., Rozhansky V. 2012 Nucl. Fusion 52054011

[20] Liu Y. Q. et al 2012 Plasma Phys. Control. Fusion 54124013

[21] Liu Y. Q. et al 2012 Phys. Plasmas 19072509

[22] Heyn M. F. et al 2008 Nucl. Fusion 48024005

[23] Finken K. H. et al 2005 Phys. Rev. Lett. 94015003

[24] Koslowski H. R. et al 2006 Nucl. Fusion 46 L1

[25] Bock M. F. M. D. et al 2008 Nucl. Fusion 48015007

[26] Yu Q. et al 2009 Phys. Plasmas 16042301

[27] Yu Q., Gunter S. 2009 Nucl. Fusion 49062001

[28] Zhuang G. et al 2011 Nucl. Fusion 51094020

[29] Rao B. et al 2013 Rev. Sci. Instrum. 84043504

[30] Rao B. et al 2012 IEEE Transactions on Applied Superconductivity 224201804

[31] Hu Q. et al 2012 Nucl. Fusion 52083011

[32] Rao B. et al 2013 Physics Letters A 377315

[33] Jin W. et al 2013 Plasma Phys. Control. Fusion 55035010

[34] Zhuang G. et al 2013 Nucl. Fusion accepted

[35] Rao B. et al 2013 Fusion Eng. Des. Introduction to resonant magnetic perturbation coils of J-TEXT tokamak submitted

[36] Gao L. et al 2012 Rev. Sci. Instrum. $8310 \mathrm{E} 303$

[37] Yang Z. J. et al 2012 Rev. Sci. Instrum. 8310 E313

[38] Yu Q. et al 2008 Nucl. Fusion 48024007

[39] Yu Q. et al 2003 Phys. Plasmas 10797

[40] Yu Q., Gunter S. 2008 Nucl. Fusion 48065004

[41] Yu Q. 2006 Phys. Plasmas 13062310 